\title{
Phosphine-Free Palladium-Catalyzed Direct C-3 Arylation of 2-Phenylimidazo[1,2-a]pyridine Using Silver(I) Carboxylate
}

\author{
Sridevi Kona, ${ }^{1}$ Rama Suresh Ravi, ${ }^{1}$ Manab Chakravarty, ${ }^{2}$ and Venkata N. R. Chava ${ }^{3}$ \\ ${ }^{1}$ School of Chemistry, University of Hyderabad, Hyderabad 500046, India \\ ${ }^{2}$ Department of Chemistry, Birla Institute of Technology and Sciences Pilani, Hyderabad Campus, Shamirpet, \\ Hyderabad 500078, India \\ ${ }^{3}$ AG and SG Siddhartha Degree Arts and Science College, Vuyyuru, Andhra Pradesh 521165, India
}

Correspondence should be addressed to Manab Chakravarty; manabhere@yahoo.co.in

Received 24 June 2012; Accepted 5 August 2012

Academic Editor: Samir Messaoudi

Copyright (C) 2013 Sridevi Kona et al. This is an open access article distributed under the Creative Commons Attribution License, which permits unrestricted use, distribution, and reproduction in any medium, provided the original work is properly cited.

Phosphine-free palladium-catalyzed direct arylation of 2-phenyl-imidazo[1,2-a]pyridine has been developed with the concept of using silver(I) carboxylate. This protocol efficiently catalyzes the $\mathrm{C}-\mathrm{H}$ arylation of 2-phenyl-imidazo[1,2-a]pyridine with aryl iodides to afford the corresponding 2-phenyl-3-aryl-imidazo[1,2-a]pyridines in moderate to-good yields.

\section{Introduction}

Syntheses of several heterocycles via palladium-catalyzed direct $\mathrm{C}-\mathrm{H}$ bond activation using aryl halides are of great importance in recent years along with time-honored coupling reactions where expensive and toxic phosphines are widely used [1-6]. Hence, there are significant efforts to perform reactions without using any phosphines for the same reasons. In this aspect, phosphine-free palladium-catalyzed synthesis of free $(\mathrm{N}-\mathrm{H})$ as well as substituted indole and pyrroles are reported in the literature [7-9]. In 2008, Igor Larosa reported the intrinsic idea of using the several different types of $\mathrm{Ag}(\mathrm{I})$ carboxylate salts (generated in situ by the combination of $\mathrm{Ag}_{2} \mathrm{O}$ /differently substituted benzoic acids) along with $\mathrm{Pd}(\mathrm{OAc})_{2}$ to undergo $\mathrm{C}-\mathrm{H}$ bond activation under very mild conditions to afford C-2 arylated indoles with different functionalities [9]. This paper prompted us to use the same approach for the highly demanding arylation for the C3 position of imidazo[1,2-a]pyridine, a heterocycle with outstanding biological activities [10-15]. The most well known drugs like zolimidine (A, antiulcer), alpidem (B, anxiolytic), and zolpidem (C, hypnotic) [14] (Figure 1) also bear this imidazo[1,2-a]pyridine skeleton.
It is to be noted that, in 2007, the research group of Dalibor reported the phosphine-free $\mathrm{Pd}(\mathrm{OAc})_{2}$ catalyzed direct $\mathrm{C}$-arylation of free $(-\mathrm{NH})$ indoles and pyrroles in the presence of CsOAC [8]. Recently there are few reports on the Pd-catalyzed direct C-3 arylation of imidazo[1,2-a]pyridines using phosphines as ligands [16-19] along with the traditional coupling reactions where the starting material should be 3-haloimidazo[1,2-a]pyridines [20-23]. During the preparation of our paper, $\mathrm{Fu}$ and collaborators published the phosphine-free Pd-catalyzed direct arylation of imidazo [1,2-a]pyridine using KOAc as base [24]. However, prior to the work published by $\mathrm{Fu}$ et al. to the best of our knowledge, there was no report on the phosphine-free direct arylation of imidazo[1,2-a]pyridine using palladium as catalyst. In this present work, we describe a new convenient methodology for the phosphine-free direct C3 alkylation of 2-phenylimidazo[1,2-a]pyridine by the reaction of aryl iodides in the presence of $\mathrm{Pd}(\mathrm{OAc})_{2}$ as catalyst with silver(I) carboxylate which is assumed to increase the rate of the palladation step in the catalytic cycle along with its basic nature needed at the reductive elimination step [9]. This methodology also offer a new route for the direct arylation of 2-phenylimidazo[1,2-a]pyridine with aryl iodides. 


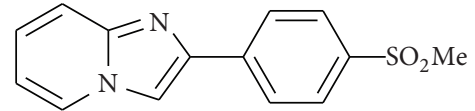

A

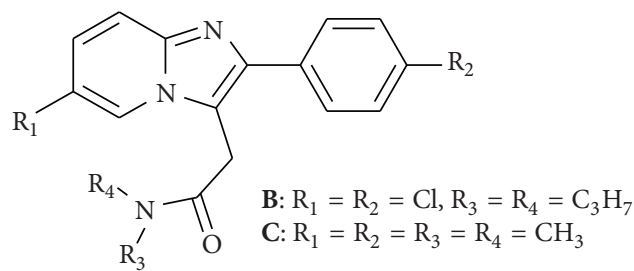

FIgURE 1: Examples of well-known drugs containing imidazo[1,2-a]pyridine.

\section{Experimental}

2.1. General. All the chemicals used were purchased from Aldrich Chemical Co. and were used without further purification. Freshly distilled solvents were used. For TLC, aluminum plates coated with silica gel containing F254 indicator were used and the spots were visualized by UV light and/or by exposing to iodine. Column chromatography was performed on silica gel 100-200 mesh, using EtOAc and hexanes mixture as eluent. The ${ }^{1} \mathrm{H}$ and ${ }^{13} \mathrm{C}$ NMR spectra were recorded using $5 \mathrm{~mm}$ tubes on a Bruker $500 \mathrm{MHz}$ NMR spectrometer (field strengths: 500 and $125 \mathrm{MHz}$, resp.,) or 400 $\mathrm{MHz}$ NMR spectrometer (field strengths: 400 and $100 \mathrm{MHz}$ resp.,) in $\mathrm{CDCl}_{3}$ solution (unless specified otherwise) with shifts referenced to $\mathrm{SiMe}_{4}\left({ }^{1} \mathrm{H},{ }^{13} \mathrm{C}: \delta=0\right)$. All $J$ values were in Hz. IR spectra were recorded on a JASCO FT/IR 5300 spectrometer. Elemental (C, H, N) analysis was done using Perkin-Elmer 240C CHN FLASH EA analyzer. Melting points were determined by using a SUPERFIT hot-stage melting point apparatus and are uncorrected.

\subsection{General Procedure for the Synthesis of 2-Phenylimida-}

$z o[1,2$-a]pyridine(1). To the solution of 2-aminopyridine $(5 \mathrm{mmol})$ in ethanol $(30 \mathrm{~mL}), 2$-bromo acetophenone $(5 \mathrm{mmol})$ was added at room temperature and the reaction mixture was heated under reflux for $3 \mathrm{~h}$. The resulting white solid was filtered off, dried, and used without further purification $[25,26]$.

2.3. General Procedure for the Direct Arylation of 2Phenylimidazo[1,2-a]pyridine. A mixture of 2-phenylimidazo[1,2-a]pyridine (194 mg, $1.0 \mathrm{mmol})$, iodobenzene $(224 \mu \mathrm{L}$, $2.0 \mathrm{mmol}), \mathrm{Pd}(\mathrm{OAc})_{2}$ (11.2 mg, $\left.5 \mathrm{~mol} \%\right), \mathrm{Ag}_{2} \mathrm{O}$ (174 mg, $0.75 \mathrm{mmol}$ ), and 2-nitrobenzoic acid (251 mg, $1.5 \mathrm{mmol})$ was stirred at $120^{\circ} \mathrm{C}$ for $12 \mathrm{~h}$ under nitrogen in DMF. The reaction mixture was filtered through a plug of silica gel and then evaporated to dryness under reduced pressure. The crude product was purified by column chromatography (Hexane: EtOAc $80: 20)$ to afford 2,3-diphenylimidazo[1,2-a]pyridine. For the synthesis of compounds $\mathbf{3 b} \mathbf{b}$ : : similar molar quantities of the respective aryl iodides were used with $1.0 \mathrm{mmol}$ of 2 phenylimidazo[1,2-a]pyridine.

2,3-Diphenylimidazo[1,2-a]pyridine (3a). Colorless solid, Yield $82 \%, 0.22 \mathrm{~g}, \mathrm{Mp} 150-152^{\circ} \mathrm{C} ;{ }^{1} \mathrm{H} \mathrm{NMR}(400 \mathrm{MHz}$, $\left.\mathrm{CDCl}_{3}\right): \delta_{\mathrm{H}} 6.75\left(\mathrm{t},{ }^{3} J(\mathrm{H}-\mathrm{H})=6.4 \mathrm{~Hz}, 1 \mathrm{H}, \mathrm{Ar}-\mathrm{H}\right), 7.20-7.31$ (m, $4 \mathrm{H}$, Ar- $H$ ), 7.46-7.56 (m, 5H, Ar-H), 7.68-7.71 (m, 3H,
Ar- $H), 7.97\left(\mathrm{~d},{ }^{3} J(\mathrm{H}-\mathrm{H})=6.8 \mathrm{~Hz}, 1 \mathrm{H}\right.$, Ar- $\left.H\right) .{ }^{13} \mathrm{C} \mathrm{NMR}$ $\left(100 \mathrm{MHz}, \mathrm{CDCl}_{3}\right): \delta_{\mathrm{C}} \cdot 112.4,117.5,120.0,123.3,124.8$, $127.6,128.1,128.3,129.0,129.6,129.8,130.8,134.0,137.4$, 144.7. IR $\left(\nu_{\max }, \mathrm{cm}^{-1}\right): 2924,1506,1346,754,698 . \mathrm{LC} / \mathrm{MS}$, $m / z 271[\mathrm{M}+1]^{+}$; Anal. Calcd. for $\mathrm{C}_{19} \mathrm{H}_{14} \mathrm{~N}_{2}$ : C, 84.42; $\mathrm{H}$, 5.22; N, 10.36\%. Found: C, 84.31; H, 5.18; N, 10.26\%.

2-Phenyl-3-p-tolylimidazo[1,2-a]pyridine (3b). Colorless solid, Yield 81\%, $0.23 \mathrm{~g}, \mathrm{Mp} 126-128^{\circ} \mathrm{C} ;{ }^{1} \mathrm{H}$ NMR $(400 \mathrm{MHz}$, $\left.\mathrm{CDCl}_{3}\right): \delta_{\mathrm{H}} 2.47\left(\mathrm{~s}, 3 \mathrm{H}, \mathrm{CH}_{3}\right), 6.72\left(\mathrm{t},{ }^{3} \mathrm{~J}(\mathrm{H}-\mathrm{H})=6.0 \mathrm{~Hz}, 1 \mathrm{H}\right.$, Ar-H), 7.17-7.34 (m, 8H, Ar-H), 7.67-7.71 (m, 3H, Ar-H), $7.95\left(\mathrm{~d},{ }^{3} \mathrm{~J}(\mathrm{H}-\mathrm{H})=6.8 \mathrm{~Hz}, 1 \mathrm{H}, \operatorname{Ar}-H\right) .{ }^{13} \mathrm{C}$ NMR $(100 \mathrm{MHz}$, $\left.\mathrm{CDCl}_{3}\right): \delta_{\mathrm{C}} .21 .4,112.1,117.5,121.1,123.3,124.5,126.8$, $127.4,128.1,128.3,130.3,130.5,134.3,138.9,142.2,144.8$. IR $\left(v_{\max }, \mathrm{cm}^{-1}\right): 2924,1506,1346,754,698$. LC/MS, $m / z 285$ $[\mathrm{M}+1]^{+}$; Anal. Calcd. for $\mathrm{C}_{20} \mathrm{H}_{16} \mathrm{~N}_{2}: \mathrm{C}, 84.48 ; \mathrm{H}, 5.67 ; \mathrm{N}$, 9.85\%. Found: C, 84.36; H, 5.61; N, 9.96\%.

2-Phenyl-3-m-tolylimidazo[1,2-a]pyridine (3c). Colorless solid, Yield 78\%, $0.22 \mathrm{~g}, \mathrm{Mp} 156-158^{\circ} \mathrm{C} ;{ }^{1} \mathrm{H}$ NMR $(400 \mathrm{MHz}$, $\left.\mathrm{CDCl}_{3}\right): \delta_{\mathrm{H}} 2.41\left(\mathrm{~s}, 3 \mathrm{H}, \mathrm{CH}_{3}\right), 6.73\left(\mathrm{t},{ }^{3} J(\mathrm{H}-\mathrm{H})=6.4 \mathrm{~Hz}\right.$, $1 \mathrm{H}, \operatorname{Ar}-H), 7.18-7.44(\mathrm{~m}, 8 \mathrm{H}, \operatorname{Ar}-H), 7.67-7.71(\mathrm{~m}, 3 \mathrm{H}$, Ar- $H), 7.95\left(\mathrm{~d},{ }^{3} J(\mathrm{H}-\mathrm{H})=6.4 \mathrm{~Hz}, 1 \mathrm{H}, \mathrm{Ar}-H\right) .{ }^{13} \mathrm{C}$ NMR $(100$ $\left.\mathrm{MHz}, \mathrm{CDCl}_{3}\right): \delta_{\mathrm{C}} .21 .5,112.2,117.5,121.3,123.4,124.6$, $127.4,127.9,128.0,128.3,129.5,129.7,129.8,131.2,134.3$, 139.3, 142.2, 144.7. IR $\left(\nu_{\max }, \mathrm{cm}^{-1}\right): 2924,1604,1505,1385$, 1342, 754, 704. LC/MS, $m / z 285[\mathrm{M}+1]^{+}$; Anal. Calcd. for $\mathrm{C}_{20} \mathrm{H}_{16} \mathrm{~N}_{2}$ : C, 84.48; H, 5.67; N, 9.85\%. Found: C, 84.38; H, $5.63 ; \mathrm{N}, 9.79 \%$. X-ray analysis was done for this sample.

3-(3-Nitrophenyl)-2-Phenylimidazo[1, 2-a]pyridine (3d). Yellow colored solid, Yield 90\%, 0.28 g, Mp 158-160 C; ${ }^{1} \mathrm{H}$ NMR $\left(500 \mathrm{MHz} \mathrm{CDCl}_{3}\right): \delta_{\mathrm{H}} 6.68\left(\mathrm{td},{ }^{3} J(\mathrm{H}-\mathrm{H})=7.0 \mathrm{~Hz}, 1.5 \mathrm{~Hz}\right.$, $1 \mathrm{H}, \mathrm{Ar}-\mathrm{H}), 7.28-7.33$ (m, 4H, Ar- H), 7.60-7.80 (m, 5H, ArH), $8.02\left(\mathrm{~d},{ }^{3} J(\mathrm{H}-\mathrm{H})=6.5 \mathrm{~Hz}, 1 \mathrm{H}, \mathrm{Ar}-\mathrm{H}\right), 8.34-8.36(\mathrm{~m}$, $1 \mathrm{H}, \mathrm{Ar}-\mathrm{H}), 8.42\left(\mathrm{t},{ }^{3} \mathrm{~J}(\mathrm{H}-\mathrm{H})=2.0 \mathrm{~Hz}, 1 \mathrm{H}, \mathrm{Ar}-H\right) .{ }^{13} \mathrm{C} \mathrm{NMR}$ $\left(125 \mathrm{MHz} \mathrm{CDCl}_{3}\right): \delta_{\mathrm{C}} .112 .2,117.1,117.8,121.8,123.7$, $124.6,127.2,127.5,127.6,130.1,132.5,135.8,143.6,144.8$, 146.5. IR $\left(\nu_{\max }, \mathrm{cm}^{-1}\right): 2924,1506,1346,754,698 . \mathrm{LC} / \mathrm{MS}$, $m / z 316[\mathrm{M}+1]^{+}$; Anal. Calcd. for $\mathrm{C}_{19} \mathrm{H}_{13} \mathrm{~N}_{3} \mathrm{O}_{2}$ : C, 72.37; $\mathrm{H}$, 4.16; N, 13.33\%. Found: C, 72.45; H, 4.08; N, 13.45\%.

1-(4-(2-Phenyllimidazo[1, 2-a]pyridine-3-yl)phenyl)ethanone (3e). Colorless solid, Yield 85\%, 0.26 g, Mp $122-124{ }^{\circ} \mathrm{C} ;{ }^{1} \mathrm{H}$ NMR $\left(400 \mathrm{MHz}, \mathrm{CDCl}_{3}\right): \delta_{\mathrm{H}} 2.67\left(\mathrm{~s}, 3 \mathrm{H}, \mathrm{CH}_{3}\right), 6.79(\mathrm{t}$, $\left.{ }^{3} J(\mathrm{H}-\mathrm{H})=6.8 \mathrm{~Hz}, 1 \mathrm{H}, \operatorname{Ar}-H\right), 7.22-7.30(\mathrm{~m}, 4 \mathrm{H}, \mathrm{Ar}-\mathrm{H})$, 7.56-7.63 (m, 4H, Ar-H), $7.71\left(\mathrm{~d},{ }^{3} J(\mathrm{H}-\mathrm{H})=1.6 \mathrm{~Hz}, 1 \mathrm{H}, \mathrm{Ar}-\right.$ $H), 8.04\left(\mathrm{~d},{ }^{3} J(\mathrm{H}-\mathrm{H})=6.8 \mathrm{~Hz}, 1 \mathrm{H}, \mathrm{Ar}-H\right), 8.09\left(\mathrm{~d},{ }^{3} J(\mathrm{H}-\mathrm{H})=\right.$ $8.0 \mathrm{~Hz}, 2 \mathrm{H}, \mathrm{Ar}-H) .{ }^{13} \mathrm{C} \mathrm{NMR}\left(100 \mathrm{MHz}^{\mathrm{C}} \mathrm{CDCl}_{3}\right): \delta_{\mathrm{C}} \cdot 26.7$, 
$112.7,117.9,119.9,123.1,125.2,127.9,128.4,129.5,130.6$, 133.8, 134.8, 137.0, 143.6, 145.3. IR $\left(\nu_{\max }, \mathrm{cm}^{-1}\right): 3053,1684$, $1605,1267,958,698 \mathrm{LC} / \mathrm{MS}, \mathrm{m} / z 313[\mathrm{M}+1]^{+}$; Calcd. for $\mathrm{C}_{21} \mathrm{H}_{16} \mathrm{~N}_{2} \mathrm{O}$ : C, 80.75; H, 5.16; N, 8.97\%. Found: C, 80.65; H, $5.21 ; \mathrm{N}, 8.86 \%$.

2-Phenyl-3-(3,4,5-trimethoxyphenyl)imidazo[1, 2-

a]pyridine (3f). Colorless solid, Yield 86\%, $0.31 \mathrm{~g}, \mathrm{Mp}$ $122-124^{\circ} \mathrm{C} ;{ }^{1} \mathrm{H}$ NMR $\left(400 \mathrm{MHz}, \mathrm{CDCl}_{3}\right): \delta_{\mathrm{H}} 3.80(\mathrm{~s}, 6 \mathrm{H}$, $\left.\left(\mathrm{OCH}_{3}\right)_{2}\right), 3.97\left(\mathrm{~s}, 3 \mathrm{H}, \mathrm{OCH}_{3}\right), 6.66(\mathrm{~s}, 2 \mathrm{H}, \mathrm{Ar}-\mathrm{H}), 6.75-6.78$ (m, 1H, Ar-H), 7.19-7.34 (m, 4H, Ar-H), 7.67-7.75 (m, 3H, Ar-H), $7.98\left(\mathrm{dd},{ }^{3} J(\mathrm{H}-\mathrm{H})=0.8 \mathrm{~Hz}, 6.8 \mathrm{~Hz}, 1 \mathrm{H}, \mathrm{Ar}-H\right)$. ${ }^{13} \mathrm{C}$ NMR $\left(100 \mathrm{MHz}, \mathrm{CDCl}_{3}\right): \delta_{\mathrm{C}} .56 .3,61.1,107.7,112.3$, $117.6,121.1,123.5,124.6,125.2,127.5,127.9,128.3,134.1$, $138.5,142.1,144.7,154.2$. IR $\left(v_{\max }, \mathrm{cm}^{-1}\right): 2930,1579,1238$, 1120, 736, 698. LC/MS, $m / z 361[\mathrm{M}+1]^{+}$; Anal. Calcd. for $\mathrm{C}_{22} \mathrm{H}_{20} \mathrm{~N}_{2} \mathrm{O}_{3}$ : C, 73.32; H, 5.59; N, 7.77\%. Found: C, 73.45; $\mathrm{H}, 5.52 ; \mathrm{N}, 7.85 \%$.

2-Phenyl-3-o-tolylimidazo[1,2-a]pyridine (3g). Colorless solid, Yield 51\%, 0.14 g, Mp 124-126 C; ${ }^{\circ} \mathrm{H}$ NMR $(500 \mathrm{MHz}$, $\left.\mathrm{CDCl}_{3}\right): \delta_{\mathrm{H}} 2.48\left(\mathrm{~s}, 3 \mathrm{H}, \mathrm{CH}_{3}\right), 6.73\left(\mathrm{td},{ }^{3} \mathrm{~J}(\mathrm{H}-\mathrm{H})=7.0 \mathrm{~Hz}\right.$, $1.0 \mathrm{~Hz}, 1 \mathrm{H}, \mathrm{Ar}-H), 7.19-7.36(\mathrm{~m}, 8 \mathrm{H}, \mathrm{Ar}-H), 7.69-7.72(\mathrm{~m}$, $3 \mathrm{H}, \mathrm{Ar}-\mathrm{H}), 7.96\left(\mathrm{~d},{ }^{3} J(\mathrm{H}-\mathrm{H})=7.0 \mathrm{~Hz}, 1 \mathrm{H}, \mathrm{Ar}-H\right) .{ }^{13} \mathrm{C} \mathrm{NMR}$ $\left(100 \mathrm{MHz}, \mathrm{CDCl}_{3}\right): \delta_{\mathrm{C}} \cdot 21.5,112.2,117.5,121.3,123.4,124.7$, $127.5,127.9,128.0,128.3,129.5,129.7,131.2,134.1,139.3$, 142.1, 144.7. IR $\left(\nu_{\max }, \mathrm{cm}^{-1}\right): 3021,1523,1378,1351,752$, 612. LC/MS, $m / z 285[\mathrm{M}+1]^{+}$; Anal. Calcd. for $\mathrm{C}_{20} \mathrm{H}_{16} \mathrm{~N}_{2}$ : C, 84.48; H, 5.67; N, 9.85\%. Found: C, 84.58; H, 5.61; N, $9.75 \%$.

3-(4-Nitrophenyl)-2-phenylimidazo[1, 2-a]pyridine (3h). Yellow colored solid, Yield 89\%, 0.28 g, Mp 150-152 ${ }^{\circ}$; ${ }^{1} \mathrm{H}$ NMR $\left(500 \mathrm{MHz}, \mathrm{CDCl}_{3}\right): \delta_{\mathrm{H}} 6.86-6.88(\mathrm{~m}, 1 \mathrm{H}, \mathrm{Ar}-\mathrm{H}), 7.28-7.36$ (m, 4H, Ar-H), 7.59-7.60 (m, 2H, Ar- H), $7.68\left(\mathrm{~d},{ }^{3} J(\mathrm{H}-\mathrm{H})=\right.$ $9.0 \mathrm{~Hz}, 2 \mathrm{H}, \mathrm{Ar}-H), 7.75\left(\mathrm{~d},{ }^{3} J(\mathrm{H}-\mathrm{H})=9.0 \mathrm{~Hz}, 1 \mathrm{H}, \mathrm{Ar}-H\right), 8.11$ $\left(\mathrm{d},{ }^{3} J(\mathrm{H}-\mathrm{H})=7.0 \mathrm{~Hz}, 1 \mathrm{H}, \operatorname{Ar}-\mathrm{H}\right), 8.38\left(\mathrm{~d},{ }^{3} J(\mathrm{H}-\mathrm{H})=8.5 \mathrm{~Hz}\right.$, $2 \mathrm{H}, \mathrm{Ar}-\mathrm{H}) .{ }^{13} \mathrm{C} \mathrm{NMR}\left(125 \mathrm{MHz}, \mathrm{CDCl}_{3}\right): \delta_{\mathrm{C}} \cdot 113.2,118.1$, $118.8,122.8,124.7,125.6,128.2,128.5,128.6,131.1,133.5$, 136.7, 144.5, 145.7, 147.5. IR $\left(\nu_{\max }, \mathrm{cm}^{-1}\right): 3063,1599,1566$, $1105,854,736$. LC/MS, $m / z 316[\mathrm{M}+1]^{+}$; Anal. Calcd. for $\mathrm{C}_{19} \mathrm{H}_{13} \mathrm{~N}_{3} \mathrm{O}_{2}$ : C, 72.37; H, 4.16; N, 13.33\%. Found: C, 72.34; $\mathrm{H}, 4.23 ; \mathrm{N}, 13.21 \%$.

3-(3,5-Dimethylphenyl)-2-diphenylimidazo[1, 2-a]pyridine (3i). Colorless solid, Yield $84 \%, 0.25 \mathrm{~g}, \mathrm{Mp} 158-160^{\circ} \mathrm{C} ;{ }^{1} \mathrm{H}$ $\left.\mathrm{NMR}\left(400 \mathrm{MHz}, \mathrm{CDCl}_{3}\right): \delta_{\mathrm{H}} 2.39\left(\mathrm{~s}, 6 \mathrm{H}, \mathrm{CH}_{3}\right)_{2}\right), 6.74(\mathrm{td}$, $\left.{ }^{3} J(\mathrm{H}-\mathrm{H})=6.4 \mathrm{~Hz}, 1.2 \mathrm{~Hz}, 1 \mathrm{H}, \operatorname{Ar}-H\right), 7.08(\mathrm{~s}, 2 \mathrm{H}, \operatorname{Ar}-H)$, 7.14 (s, 1H, Ar-H), 7.18-7.33 (m, 4H, Ar-H), 7.67-7.74 (m, $3 \mathrm{H}, \mathrm{Ar}-H), 7.94\left(\mathrm{~d},{ }^{3} \mathrm{~J}(\mathrm{H}-\mathrm{H})=6.8 \mathrm{~Hz}, 1 \mathrm{H}, \mathrm{Ar}-H\right) .{ }^{13} \mathrm{C} \mathrm{NMR}$ $\left(100 \mathrm{MHz}, \mathrm{CDCl}_{3}\right): \delta_{\mathrm{C}} .21 .4,112.1,117.4,121.4,123.5,124.5$, $127.4,127.9,128.2,128.3,129.7,130.7,134.3,139.2,142.0$, 144.7. IR $\left(\nu_{\max }, \mathrm{cm}^{-1}\right): 2920,1601,1444,1342,752,702$. LC/MS, $m / z 299[\mathrm{M}+1]^{+}$; Anal. Calcd. for $\mathrm{C}_{21} \mathrm{H}_{18} \mathrm{~N}_{2}$ : C, 84.53; H, 6.08; N, 9.39\%. Found: C, 84.45; H, 6.15; N, 9.45\%. 3-(2-Nitrophenyl)-2-phenylimidazo[1,2-a]pyridine (3j). Yellow colored solid, Yield 76\%, 0.24 g, Mp 154-156 C; ${ }^{1} \mathrm{H}$ NMR $\left(400 \mathrm{MHz}, \mathrm{CDCl}_{3}\right): \delta_{\mathrm{H}} 6.83-6.87(\mathrm{~m}, 1 \mathrm{H}, \mathrm{Ar}-\mathrm{H}), 7.27-7.35$ (m, 4H, Ar-H), 7.56-7.67 (m, 4H, Ar-H), 7.73(d, ${ }^{3} J(\mathrm{H}-\mathrm{H})$ $=8.8 \mathrm{~Hz}, 1 \mathrm{H}, \mathrm{Ar}-\mathrm{H}), 8.09\left(\mathrm{~d},{ }^{3} J(\mathrm{H}-\mathrm{H})=6.8 \mathrm{~Hz}, 1 \mathrm{H}, \mathrm{Ar}-\mathrm{H}\right)$, $8.38\left(\mathrm{~d},{ }^{3} \mathrm{~J}(\mathrm{H}-\mathrm{H})=8.5 \mathrm{~Hz}, 2 \mathrm{H}, \mathrm{Ar}-\mathrm{H}\right) .{ }^{13} \mathrm{C} \mathrm{NMR}(100 \mathrm{MHz}$,
$\left.\mathrm{CDCl}_{3}\right): \delta \mathrm{C} .113 .2,118.1,118.8,122.8,124.7,125.6,126.1$, $128.2,128.5,128.6,131.0,133.4,136.7,139.4,145.7,147.5$. IR $\left(\nu_{\max }, \mathrm{cm}^{-1}\right): 2924,1528,1352,851,750 . \mathrm{LC} / \mathrm{MS}, \mathrm{m} / \mathrm{z} 316$ $[\mathrm{M}+1]^{+}$; Anal. Calcd. for $\mathrm{C}_{19} \mathrm{H}_{13} \mathrm{~N}_{3} \mathrm{O}_{2}$ : C,72.37; $\mathrm{H}, 4.16 ; \mathrm{N}$, $13.33 \%$. Found: C, 72.41; H, 4.26; N, 13.26\%.

2-Phenyl-3-(thiophen-2-yl)imidazo[1,2-a]pyridine (3k). Yellow colored solid, Yield 66\%, 0.18 g, Mp 118-120 C; ${ }^{1} \mathrm{H}$ NMR $\left(400 \mathrm{MHz}, \mathrm{CDCl}_{3}\right): \delta_{\mathrm{H}} 6.80\left(\mathrm{~d},{ }^{3} J(\mathrm{H}-\mathrm{H})=6.8 \mathrm{~Hz}, 1 \mathrm{H}, \mathrm{Ar}-\right.$ $H), 7.23-7.33(\mathrm{~m}, 6 \mathrm{H}, \operatorname{Ar}-H), 7.60\left(\mathrm{~d},{ }^{3} J(\mathrm{H}-\mathrm{H})=4.8 \mathrm{~Hz}, 1 \mathrm{H}\right.$, Ar- $H), 7.68-7.76(\mathrm{~m}, 2 \mathrm{H}, \mathrm{Ar}-H), 7.83\left(\mathrm{~d},{ }^{3} J(\mathrm{H}-\mathrm{H})=7.2 \mathrm{~Hz}\right.$, $1 \mathrm{H}, \mathrm{Ar}-\mathrm{H}), 8.00\left(\mathrm{~d},{ }^{3} \mathrm{~J}(\mathrm{H}-\mathrm{H})=6.8 \mathrm{~Hz}, 1 \mathrm{H}, \mathrm{Ar}-\mathrm{H}\right) .{ }^{13} \mathrm{C} \mathrm{NMR}$ $\left(100 \mathrm{MHz}, \mathrm{CDCl}_{3}\right): \delta_{\mathrm{C}} 112.6,113.4,117.4,123.9,124.3,125.3$, $127.8,128.0,128.3,128.4,129.9,130.3,133.7,144.4,145.3$. IR $\left(v_{\max }, \mathrm{cm}^{-1}\right): 2957,2854,1462,1377,1261,1097,1020,804$. LC/MS, $m / z 277[\mathrm{M}+1]^{+}$; Anal. Calcd. for $\mathrm{C}_{17} \mathrm{H}_{12} \mathrm{~N}_{2} \mathrm{~S}$ : C, 73.88; H, 4.38; N, 10.14\%. Found: C, 73.80; H, 4.42; N, 10.12.

\section{Results and Discussion}

Using $\mathrm{Pd}(\mathrm{OAc})_{2}$ as catalyst and several different types of silver(I) acetates or carboxylates, we have begun phosphinefree reactions of 2-phenylimidazo[1,2-a]pyridines (1) with phenyl iodide to afford the direct C-3 arylated product 2,3diphenyl imidazo[1,2-a]pyridine (2a) (Scheme 1). We have taken this reaction as a model to optimize the yield by changing different combinations of $\mathrm{Ag}(\mathrm{I})$ carboxylate (generated in situ from $\mathrm{Ag}_{2} \mathrm{O}$ and different carboxylic acids) along with other bases and solvents (Table 1). Indeed, compound $\mathbf{1}$ is a very stable starting material that could be synthesized in a convenient way by the reaction of cheap commercially available 2-aminopyridine and 2-bromoacetophenone. Moreover, 2-phenylimidazo[1,2-a]pyridine derivatives are well established as potent and selective ligands for peripheral benzodiazepine receptor [10].

There was no progress of this reaction at room temperature. The highly coordinating dimethylformamide (DMF) was the best choice as a solvent for the different combination of $\mathrm{Ag}(\mathrm{I})$ salt. The more acidic $\mathrm{o}$-nitro benzoic acid was more effective combination than other acids with $\mathrm{Ag}_{2} \mathrm{O}$ for the above reaction to afford compound 2a with high yield in $\mathrm{DMF}$ at $120^{\circ} \mathrm{C}$ under nitrogen. To find out the extent of this reaction, we have used several aryl iodides substituted with both electron withdrawing and donating groups for this reaction. The products and yields are pointed out in Table 2 .

It is experiential that the percentages of yields do not vary considerably (entries $2,4,5$, and 6) with the type of substiutents present (or absent; entry 1 ) in the phenyl ring of aryl iodides. Both, electron-withdrawing (entries 4, 5, 8, and 10) and electron-donating substitutents (entries 2, 3, 6, 7, and 9) show the same result. As expected, the more hindered ortho-substituted aryl iodides react considerably more slowly than the meta- and para-counterparts to afford slightly lower yields (entries 7 and 10). Further, we also have checked the reactivity of 2-iodothiophene as a heteroaryl iodide under the same reaction condition which affords the compound $\mathbf{3 k}$ with $66 \%$ yield. All the compounds (3a-k) were characterized by IR, NMR, mass spectroscopy, and elemental analysis. In the ${ }^{1} \mathrm{H}-\mathrm{NMR}$ spectra all aromatic protons resonate in the 


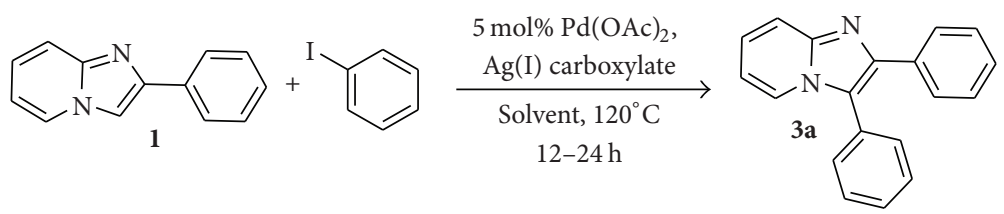

Scheme 1: $\mathrm{Pd}(\mathrm{OAc})_{2}$ catalyzed reaction of 2-phenylimidazo[1,2-a]pyridine with aryl iodides in the presence of Ag(I) carboxylate salts.

TABLE 1: Optimization of the coupling between 2-phenylimidazo[1,2-a]pyridine(1) and phenyl iodide ${ }^{\mathrm{a}}$.

\begin{tabular}{|c|c|c|c|c|}
\hline & & 2 & $\begin{array}{l}{ }^{\mathrm{Cc})_{2}}, \\
\stackrel{\mathrm{H}}{\mathrm{C}}\end{array}$ & \\
\hline Entry & Base & Acid & Solvent & Isolated yield (\%) \\
\hline 1 & $\mathrm{~K}_{2} \mathrm{CO}_{3}$ & None & DMF & Trace \\
\hline 2 & $\mathrm{~K}_{2} \mathrm{CO}_{3}$ & $\mathrm{CH}_{3} \mathrm{CO}_{2} \mathrm{H}$ & DMF & Trace \\
\hline 3 & $\mathrm{~K}_{2} \mathrm{CO}_{3}$ & $o-\mathrm{O}_{2} \mathrm{~N}-\mathrm{C}_{6} \mathrm{H}_{4}-\mathrm{CO}_{2} \mathrm{H}$ & $\mathrm{DMF}$ & Trace \\
\hline 4 & $\mathrm{Ag}_{2} \mathrm{O}$ & None & DMF & Trace \\
\hline 5 & $\mathrm{Ag}_{2} \mathrm{O}$ & $\mathrm{CH}_{3} \mathrm{CO}_{2} \mathrm{H}$ & DMF & 31 \\
\hline 6 & $\mathrm{Ag}_{2} \mathrm{O}$ & $o-\mathrm{O}_{2} \mathrm{~N}-\mathrm{C}_{6} \mathrm{H}_{4}-\mathrm{CO}_{2} \mathrm{H}$ & $\mathrm{DMF}$ & 82 \\
\hline 7 & $\mathrm{Ag}_{2} \mathrm{O}$ & $o-\mathrm{O}_{2} \mathrm{~N}-\mathrm{C}_{6} \mathrm{H}_{4}-\mathrm{CO}_{2} \mathrm{H}$ & Dioxane & 80 \\
\hline 8 & $\mathrm{Ag}_{2} \mathrm{O}$ & $o-\mathrm{O}_{2} \mathrm{~N}-\mathrm{C}_{6} \mathrm{H}_{4}-\mathrm{CO}_{2} \mathrm{H}$ & Dioxane-EtOH & 78 \\
\hline 9 & $\mathrm{Ag}_{2} \mathrm{O}$ & $\mathrm{CF}_{3} \mathrm{CO}_{2} \mathrm{H}$ & Dioxane & 38 \\
\hline
\end{tabular}

All reactions were carried out using $5 \mathrm{~mol} \% \mathrm{Pd}(\mathrm{OAc})_{2}, 1.5$ equiv (entries 1-3), or 0.75 equiv (entries $4-8$ ) of base, 1.5 equiv of acid, 1.0 equiv of 1 , and 2.0 equiv of phenyl iodide in a $0.5 \mathrm{M}$ solution, for $12 \mathrm{~h}$ at $120^{\circ} \mathrm{C}$.

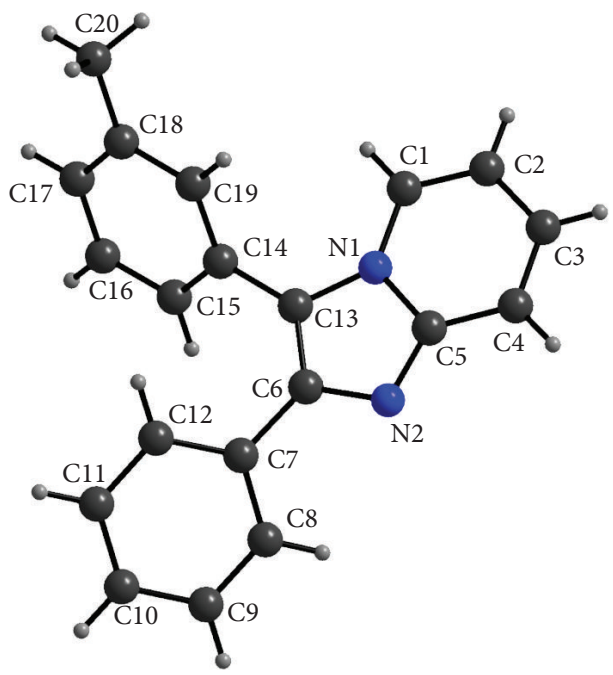

FIgURE 2: Molecular structure of the compound 3c (entry 3). (Xray data was collected on a Bruker AXS-SMART diffractometer using Mo- $_{\alpha}(\lambda=0.71073 \AA)$ radiation. The structure was solved and refined by standard methods. Crystal data for $3 \mathrm{c}: \mathrm{C}_{20} \mathrm{H}_{16} \mathrm{~N}_{2}$, $M=284.35$, Monoclinic, Space group $P 2(1) / \mathrm{n}, a=7.4312(15), b=$ $11.708(2), c=17.611(4) \AA, \alpha=90.00^{\circ}, \beta=95.36(3)^{\circ}, \gamma=90.00^{\circ}, V$ $=1525.6(5) \AA^{3}, Z=4, \mu=0.073 \mathrm{~mm}^{-1}$, data/restraints/parameters: $2677 / 0 / 200$, R indices $(I>2(I)): \mathrm{R} 1=0.0513, w R 2$ (all data) $=0.1208$. CCDC no. 885064). region $6.66-8.42 \mathrm{~Hz}$. Finally the structure for one of these compounds $3 \mathrm{c}$ is proven by $\mathrm{X}$-ray crystallography (Figure 2). (X-ray data was collected on a Bruker AXS-SMART diffractometer using Mo- $\mathrm{K}_{\alpha}(\lambda=0.71073 \AA)$ radiation. The structure was solved and refined by standard methods. Crystal data for 3c: $\mathrm{C}_{20} \mathrm{H}_{16} \mathrm{~N}_{2}, M=284.35$, Monoclinic, Space group $P 2(1) / \mathrm{n}, a=7.4312(15), b=11.708(2), c=17.611(4) \AA$, $\alpha=90.00^{\circ}, \beta=95.36(3)^{\circ}, \gamma=90.00^{\circ}, V=1525.6(5) \AA^{3}, Z=$ $4, \mu=0.073 \mathrm{~mm}^{-1}$, data/restraints/parameters: $2677 / 0 / 200, \mathrm{R}$ indices $(I>2(I)): \mathrm{R} 1=0.0513, w R 2$ (all data) $=0.1208$. CCDC no. 885064).

Selected bond lengths $(\AA)$ and angles $\left({ }^{\circ}\right)$ : N2-C5 1.323(2); N2-C6 1.369(2); N1 C5 1.395(2); C13 N1 C5 106.90(14); N2 C5 N1 110.93(15).

\section{Conclusions}

In conclusion, we have applied the catalytic $\mathrm{Pd}(\mathrm{OAc})_{2}$ and $\mathrm{Ag}(\mathrm{I})$ carboxylate combination to develop a successful methodology for this challenging direct C-3 arylation of 2-phenylimidazo[1,2-a]pyridine with differently substituted aryl iodides without the presence of phosphines or other ligands to give 3-aryl-2-phenyl imidazo[1,2-a]pyridines with moderate to-high-yield. The structure of one of these compounds has been characterized by X-ray crystallography. 
TABLE 2: Direct arylation of 2-phenylimidazo[1,2-a]pyridine(1) with differently substituted aryl iodides.

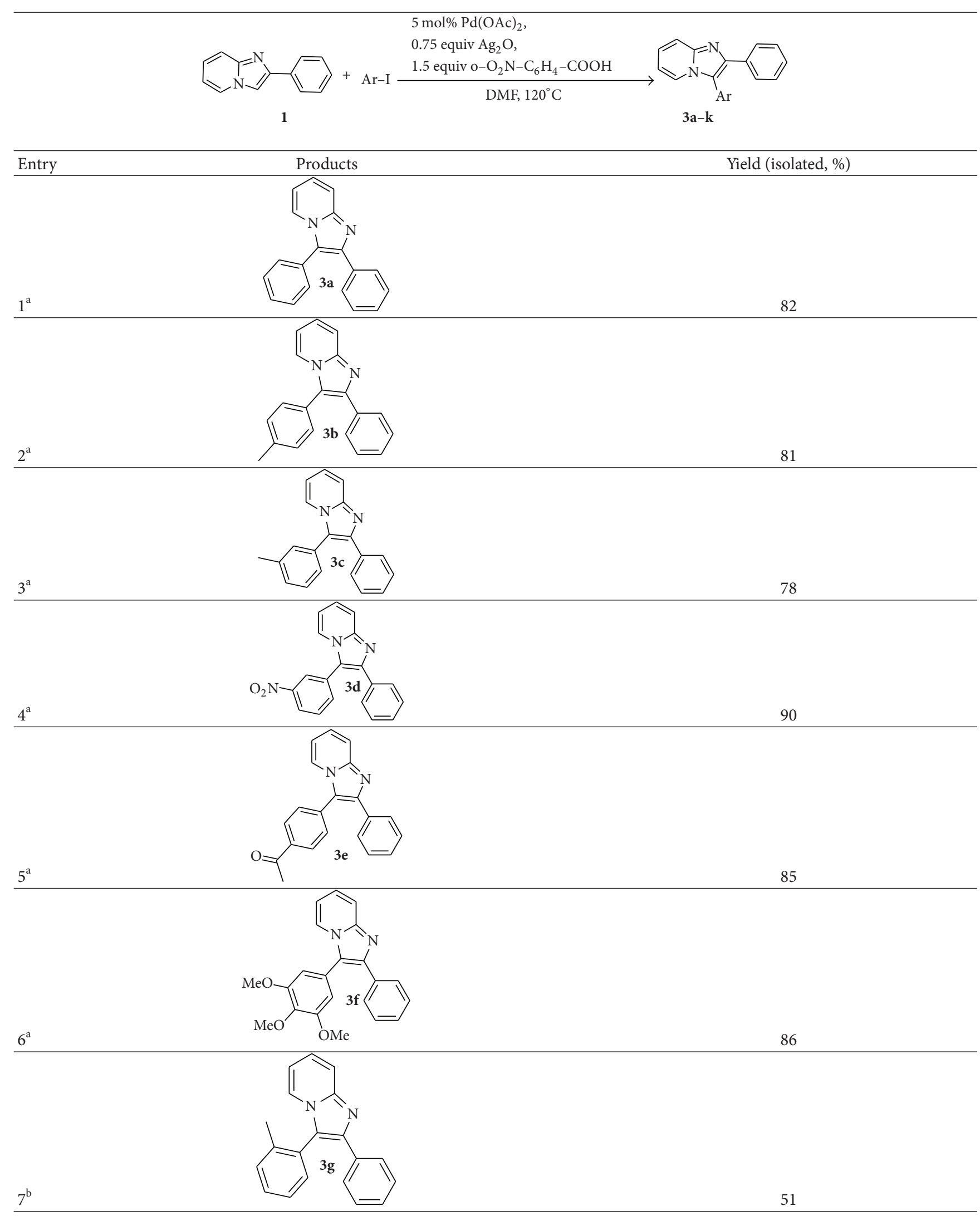


TABLE 2: Continued.

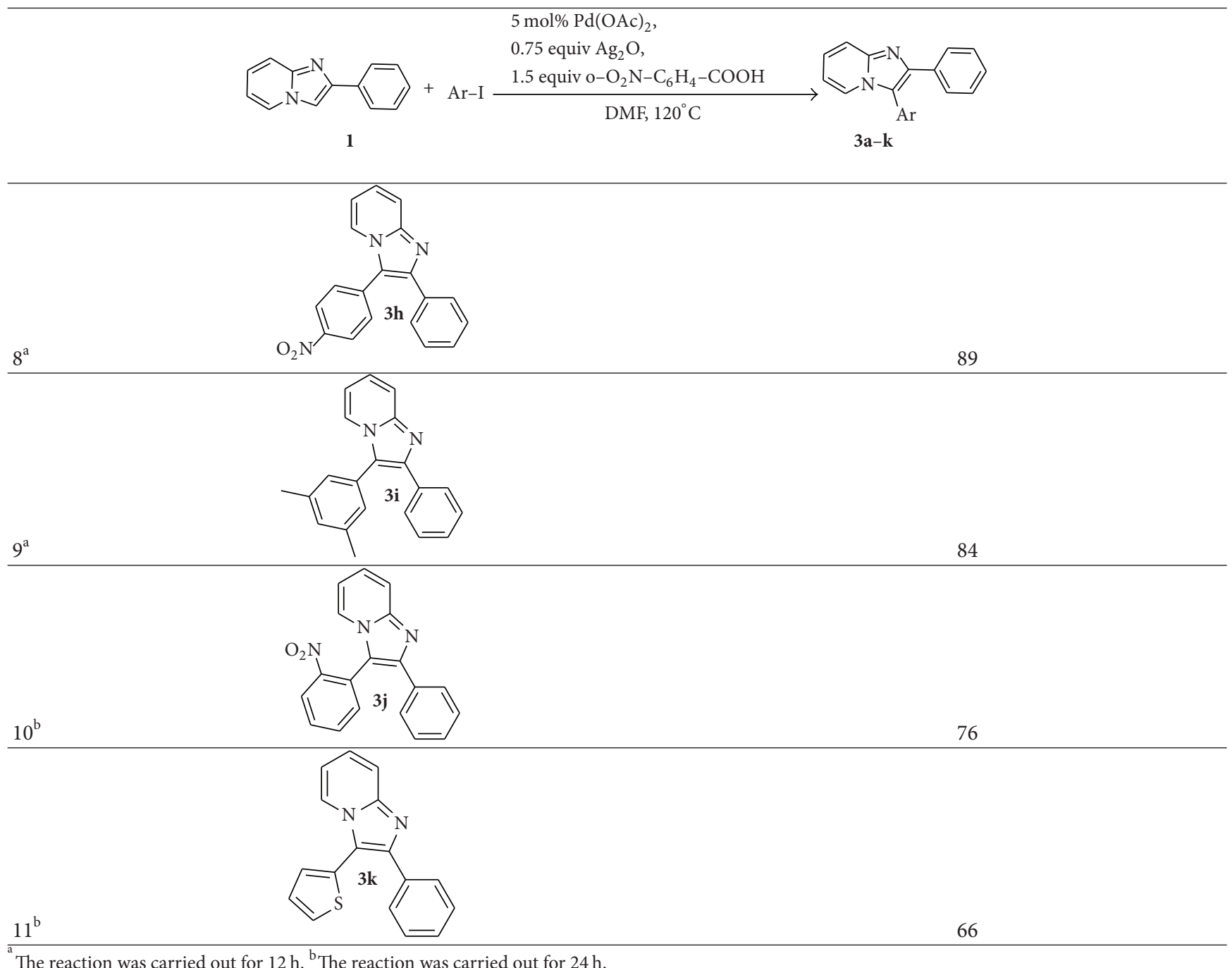

${ }^{\mathrm{a}}$ The reaction was carried out for $12 \mathrm{~h} .^{\mathrm{b}}$ The reaction was carried out for $24 \mathrm{~h}$.

\section{Acknowledgment}

S. Kona and R. Suresh thank UGC Networking Resource Centre for carrying out research work at University of Hyderabad. M. Chakravarty thanks DST-FAST Track and BITS-Pilani Hyderabad Campus for support.

\section{References}

[1] L. Ackermann and R. Vicente, "Ruthenium-catalyzed direct arylations through $\mathrm{C}-\mathrm{H}$ bond cleavages," Topics in Current Chemistry, vol. 292, pp. 211-229, 2010.

[2] O. Daugulis, H. Q. Do, and D. Shabashov, "Palladiumand copper-catalyzed arylation of carbon-hydrogen bonds," Accounts of Chemical Research, vol. 42, no. 8, pp. 1074-1086, 2009.

[3] M. Miura and M. Nomura, "Direct arylation via cleavage of activated and unactivated C-H bonds," Topics in Current Chemistry, vol. 219, pp. 211-241, 2002.

[4] D. Alberico, M. E. Scott, and M. Lautens, "Aryl-aryl bond formation by transition-metal-catalyzed direct arylation," Chemical Reviews, vol. 107, no. 1, pp. 174-238, 2007.
[5] S. Vellaisamy, M. M. Antonia, and M. J. Carlos, "Synthesis of oxygenated carbazoles by palladium-mediated oxidative double $\mathrm{C}-\mathrm{H}$ activation of diarylamines assisted by microwave irradiation," Synlett, no. 15, pp. 2375-2378, 2006.

[6] X. Chen, K. M. Engle, D. H. Wang, and J. Q. Yu, "Palladium(II)catalyzed $\mathrm{C}[\mathrm{BOND}] \mathrm{H}$ activation/C[BOND]C cross-coupling reactions: versatility and practicality," Angewandte Chemie, vol. 48, no. 28, pp. 5094-5115, 2009.

[7] M. T. Reetz and J. G. de Vries, "Ligand-free Heck reactions using low Pd-loading," Chemical Communications, no. 14, pp. 1559-1563, 2004.

[8] W. Xiang, V. G. Denis, and S. J. Dalibor, "Phosphine-free palladium-catalyzed $\mathrm{C}-\mathrm{H}$ bond arylation of free $(\mathrm{N}-\mathrm{H})$ indoles and pyrroles," Organic Chemistry, vol. 72, no. 4, pp. 1476-1479, 2007.

[9] I. J. Lebrasseur and N. Larosa, "Room temperature and phosphine free palladium catalyzed direct C-2 arylation of indoles," Journal of the American Chemical Society, vol. 130, no. 10, pp. 2926-2927, 2008.

[10] G. Trapani, M. Franco, A. Latrofa et al., "Novel 2phenylimidazo[1,2- $\alpha]$ pyridine derivatives as potent and selective ligands for peripheral benzodiazepine receptors: 
synthesis, binding affinity, and in vivo studies," Journal of Medicinal Chemistry, vol. 42, no. 19, pp. 3934-3941, 1999.

[11] Z. P. Zhuang, M. P. Kung, A. Wilson et al., "Structure-activity relationship of imidazo[1,2-a]pyridines as ligands for detecting $\beta$-amyloid plaques in the brain," Journal of Medicinal Chemistry, vol. 46, no. 2, pp. 237-243, 2003.

[12] K. S. Gudmundsson, J. D. Williams, J. C. Drach, and L. B. Townsend, "Synthesis and antiviral activity of novel erythrofuranosyl imidazo[1,2- $\alpha]$ pyridine $\mathrm{C}$-nucleosides constructed via palladium coupling of iodoimidazo $[1,2-\alpha]$ pyridines and dihydrofuran," Journal of Medicinal Chemistry, vol. 46, no. 8, pp. 1449-1455, 2003.

[13] Y. Katsura, S. Nishino, Y. Inoue, M. Tomoi, and H. Takasugi, "Studies on antiulcer drugs. II. Synthesis and antiulcer activities of imidazo[1,2- $\alpha$ ]pyridinyl-2-alkylaminobenzoxazoles and 5, 6, 7, 8-tetrahydroimidazo $[1,2-\alpha]$ pyridinyl derivatives," Chemical \& Pharmaceutical Bulletin, vol. 40, p. 371, 1992.

[14] K. J. Holm and K. L. Goa, "Zolpidem: an update of its pharmacology, therapeutic efficacy and tolerability in the treatment of insomnia," Drugs, vol. 59, no. 4, pp. 865-889, 2000.

[15] A. R. Nath and M. S. Reddy, "Models for the assignment of the chemical shifts for protons on the epoxide ring of 2,3anhydroglycopyranosides," E-Journal of Chemistry, vol. 46, no. 9, pp. 1481-1486, 2012.

[16] J. Koubachi, S. El Kazzouli, S. Berteina-Raboin, A. Mouaddib, and G. Guillaumet, "Regioselective palladium-catalyzed arylation and heteroarylation of imidazo[1,2- $\alpha]$ pyridines," Synlett, no. 19, pp. 3237-3242, 2006.

[17] B. B. Touré, B. S. Lane, and D. Sames, "Catalytic C-H arylation of SEM-protected azoles with palladium complexes of NHCs and phosphines," Organic Letters, vol. 8, no. 10, pp. 1979-1982, 2006.

[18] J. Koubachi, S. Berteina-Raboin, A. Mouaddib, and G. Guillaumet, "Pd/Cu-catalyzed oxidative $\mathrm{C}-\mathrm{H}$ alkenylation of imidazo[1,2- $\alpha$ ]pyridines," Synthesis, no. 2, pp. 271-276, 2009.

[19] J. Koubachi, S. El Kazzouli, S. Berteina-Raboin, A. Mouaddib, and G. Guillaumet, "New and efficient palladium(0)-mediated microwave-assisted direct C3 alkenylation of imidazo[1,2$\alpha$ ]pyridines," Synthesis, no. 16, Article ID Z07508SS, pp. 2537-2542, 2008.

[20] C. Enguehard, J. L. Renou, V. Collot, M. Hervet, S. Rault, and A. Gueiffier, "Reactivity of 3-iodoimidazo[1,2- $\alpha]$ pyridines using a Suzuki-type cross-coupling reaction," Journal of Organic Chemistry, vol. 65, no. 20, pp. 6572-6575, 2000.

[21] C. Enguehard, H. Allouchi, A. Gueiffier, and S. L. Buchwald, "Easy access to novel substituted 6-aminoimidazo[1,2$\alpha]$ pyridines using palladium- and copper-catalyzed aminations," Journal of Organic Chemistry, vol. 68, no. 11, pp. 4367-4370, 2003.

[22] C. Enguehard, H. Allouchi, A. Gueiffier, and S. L. Buchwald, "Ipso- or cine-substitutions of 6-haloimidazo[1,2- $\alpha]$ pyridine derivatives with different azoles depending on the reaction conditions," Journal of Organic Chemistry, vol. 68, no. 14, pp. 5614-5617, 2003.

[23] Y. Kawai, S. Satoh, H. Yamasaki, Kayakiri, K. Yoshihara, and T. Oku, "Imidazo[1,2- $\alpha]$ pyridine ring system as bone resorption inhibitors," PCT International Application, WO 9634866, 1996.

[24] H. Y. Fu, L. Chen, and H. Doucet, "Phosphine-free palladiumcatalyzed direct arylation of imidazo[1,2- $\alpha]$ pyridines with aryl bromides at low catalyst loading," The Journal of Organic Chemistry, vol. 77, no. 9, pp. 4473-4478, 2012.

[25] A. Gueiffier, M. Lhassani, A. Elhakmaoui et al., "Synthesis of acyclo-C-nucleosides in the imidazo[1,2- $\alpha]$ pyridine and pyrimidine series as antiviral agents," Journal of Medicinal Chemistry, vol. 39, no. 14, pp. 2856-2859, 1996.

[26] A. Gueiffier, S. Mavel, M. Lhassani et al., "Synthesis of imidazo[1,2- $\alpha]$ pyridines as antiviral agents," Journal of Medicinal Chemistry, vol. 41, no. 25, pp. 5108-5112, 1998. 

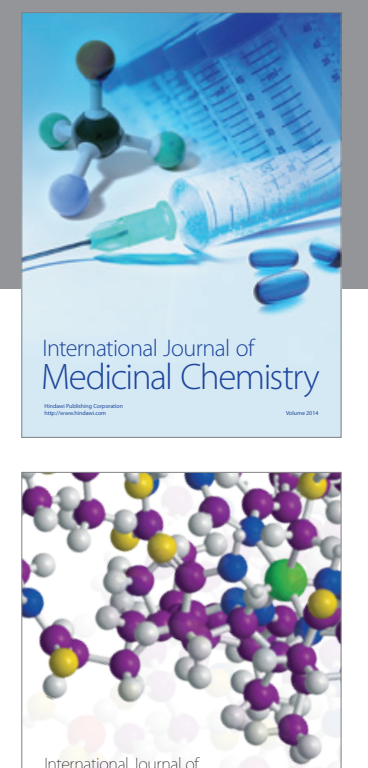

\section{Carbohydrate} Chemistry

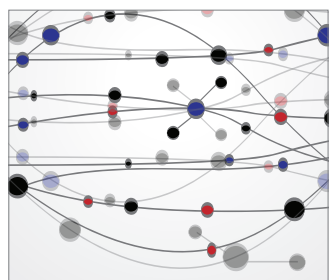

The Scientific World Journal
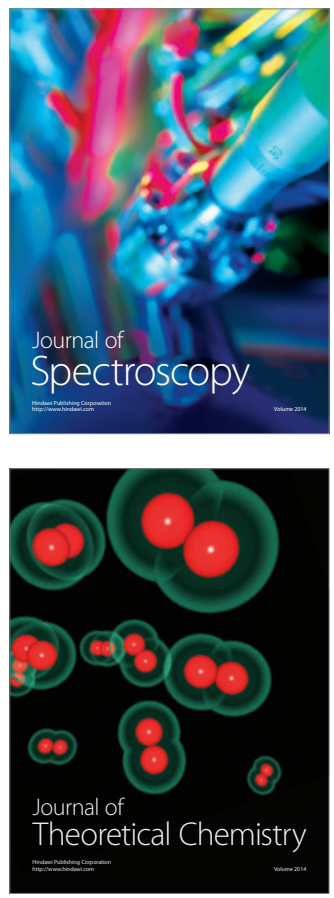
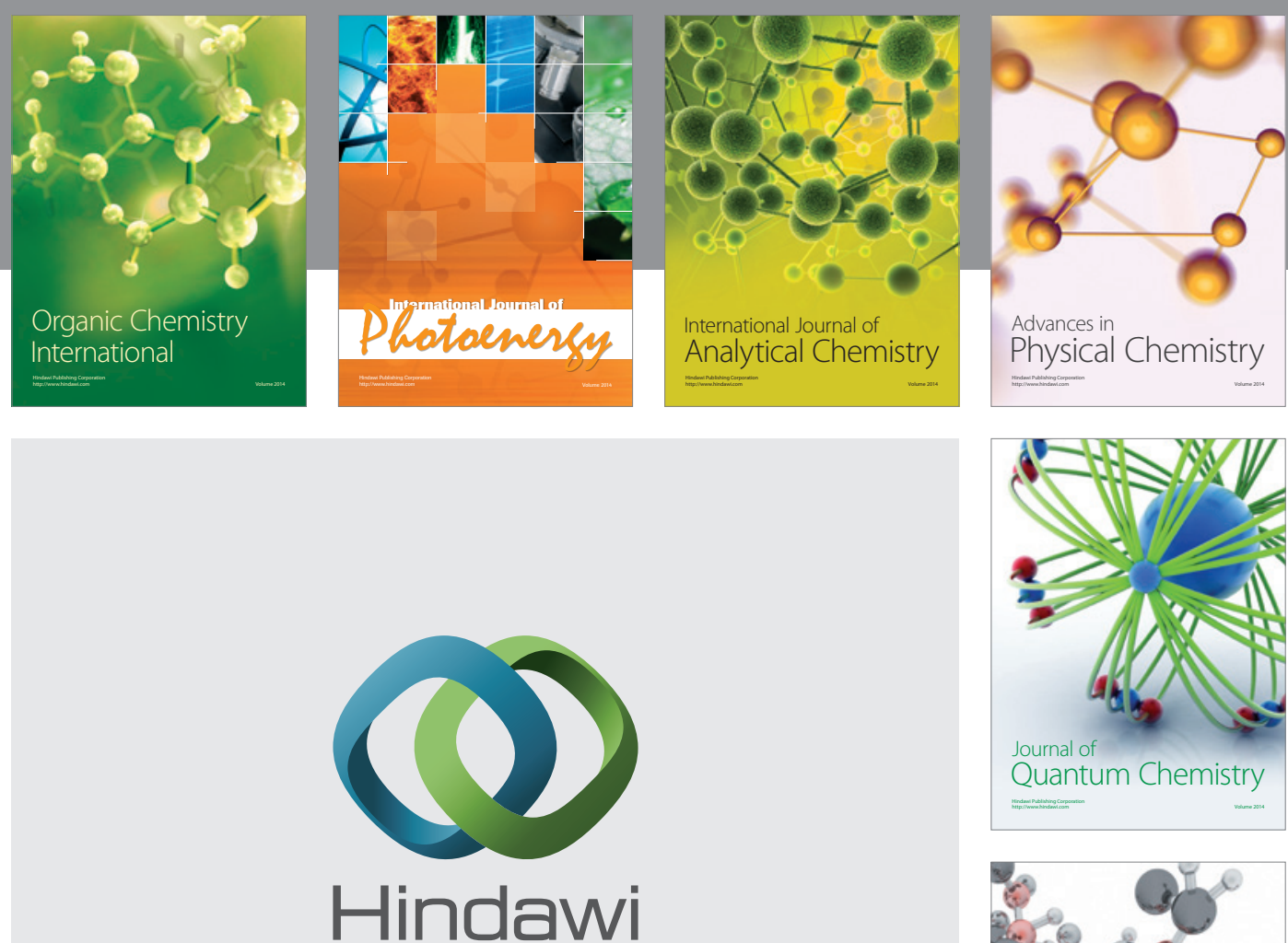

Submit your manuscripts at

http://www.hindawi.com

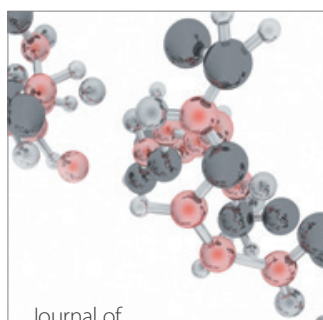

Analytical Methods

in Chemistry

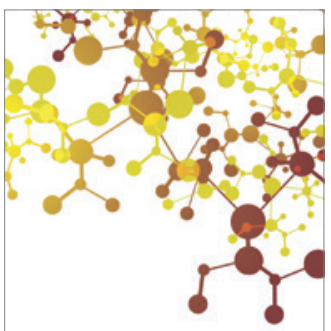

Journal of

Applied Chemistry

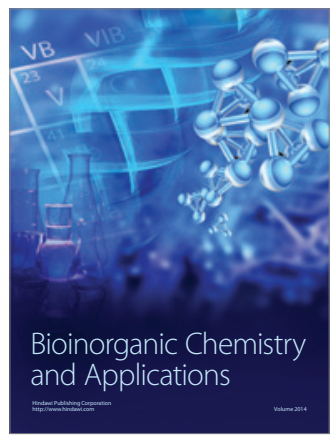

Inorganic Chemistry
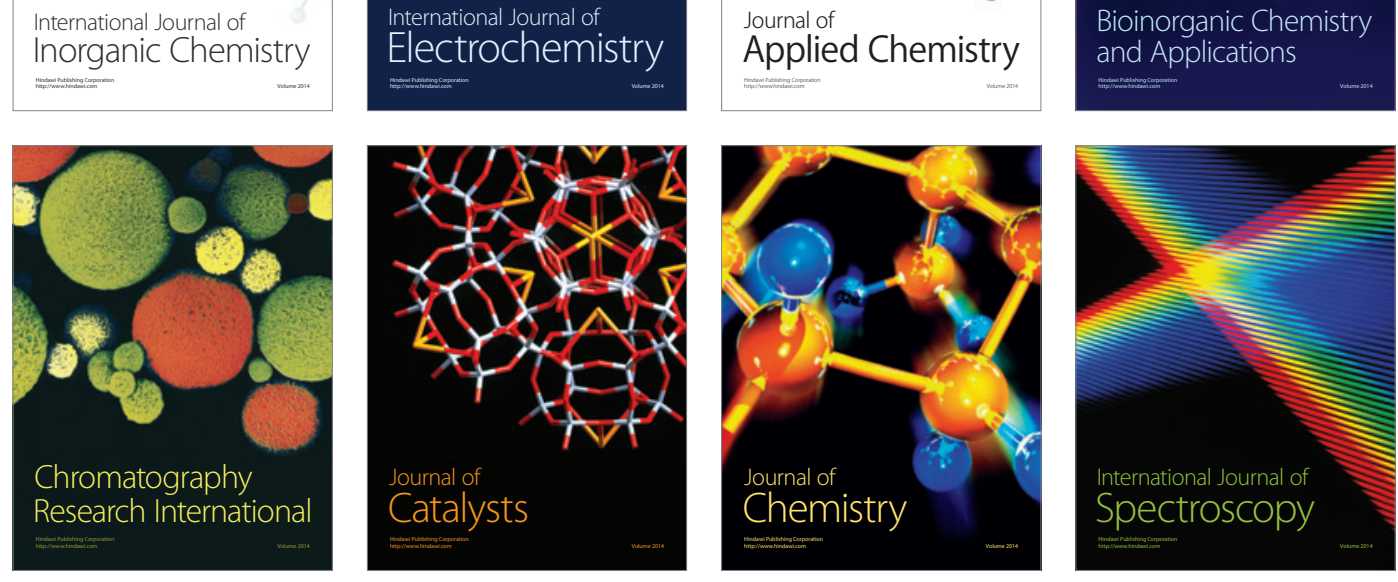